\title{
LA PHILOSOPHIE DU CORPS, ENTRE ACTION ET IDENTITÉ
}

\author{
Isabelle JOLY \\ Pesquisadora independente / Liège - Bélgica \\ isabelle.joly@emploi.belgique.be
}

Artigo submetido em fevereiro/2015 e aceito em abril/2015

DOI: 10.15628/dialektike.2015.2906

\section{RÉSUMÉ}

Le corps est un objet d'étude " multiple et un ". Nous montrerons ici la diversité des études sur le corps comme autant de points de vue possibles sur cet objet particulier et dans le même temps, nous mettrons en évidence l'unité de cette pensée qui considère un objet difficile à cerner sous un seul angle. Aujourd'hui, la philosophie du corps, fondée entre autres sur les concepts connexes de l'action et de l'identité, réunit à la fois la philosophie de l'action, la philosophie des sciences humaines et celle de l'identité entre l'esprit et le corps. Le but de cet article est de donner un aperçu général de la richesse de la philosophie contemporaine du corps.

MOTS-CLÉS : action ; sciences humaines ; identité esprit/corps.

\section{A FILOSOFIA DO CORPO, ENTRE AÇÃO E IDENTIDADE}

\section{RESUMO}

O corpo é um objeto de estudo "múltiplo e uno". Aqui mostraremos a diversidade dos estudos sobre o corpo como também os pontos de vista possíveis sobre este assunto particular. Ao mesmo tempo, evidenciaremos a unidade deste pensamento, que aprecia um objeto difícil a circunscrever sob um só ângulo. Hoje, a filosofia do corpo, com base, entre outros conceitos, sobre aqueles relacionados de ação e de identidade, traz ao mesmo tempo a filosofia da ação, a filosofia das ciências humanas e a aquela da identidade entre mente e corpo. O objetivo deste artigo é dar uma visão geral da riqueza da filosofia contemporânea do corpo.

PALAVRAS-CHAVE: ação; ciências humanas; identidade mente/corpo. 


\section{INTRODUCTION ${ }^{1}$}

La question du corps en philosophie contemporaine devrait faire à elle seule l'objet d'un texte voire d'un ouvrage. Parcourir l'ensemble de ce qui constitue une branche philosophique spécifique à l'étude du corps humain dépasse de beaucoup notre intention. La tâche est ardue même en circonscrivant celle-ci à la seule époque contemporaine. Pour paraphraser les dires du philosophe Roger Pouivet dans son livre Philosophie contemporaine, le paysage intellectuel de la philosophie contemporaine est très vaste allant de la philosophie accessible à tous jusqu'à la philosophie ésotérique destinée uniquement aux initiés. Ce même constat vaut également pour la philosophie contemporaine du corps. Nous nous bornerons ici aux grands traits de la philosophie du corps. Apparue avec le début de la philosophie, mais pourtant restée en marge, comme le souligne Michel Onfray dans ses travaux, et lui-même étant resté en dehors de l'institution universitaire, la philosophie du corps commence, toutefois, à émerger actuellement dans le paysage intellectuel « institutionnel » et cela très largement, par sa présence internationale en tant que nouvelle branche de la philosophie qui se spécialise dans l'étude du corps humain.

Sans prétendre à l'impossible exhaustivité ni à l'objectivité en ce domaine, nous décrirons cidessous les grandes lignes de quelques courants qui nous semblent s'imposer plus particulièrement aujourd'hui. Par souci de clarté, nous avons subdivisé la branche mère que nous appelons la « philosophie du corps » en trois sous-branches qui sont :

- la philosophie de l'action,

- la philosophie des sciences humaines,

- la philosophie de l'identité esprit/corps.

Chacune de ces sous-sections se répartit, de nouveau, en courants spécifiques à savoir : l'éthique, l'hédonisme, l'épistémologie, la philosophie du sexe et du genre et pour terminer, l'étude du problème de la relation entre l'esprit et le corps, qui peut s'inscrire dans trois contextes, celui de la phénoménologie, de la philosophie cognitive et de la philosophie énactive ${ }^{2}$. Cette subdivision -même très sommaire- est nécessaire, car elle met en évidence la diversité des pensées actuelles du corps. Enfin, nous tâcherons de montrer l'unité de la philosophie contemporaine du corps malgré l'apparente dispersion de la pensée sur le corps humain.

\section{LES SUBDIVISIONS DE LA PHILOSOPHIE DU CORPS. LA DOUBLE DISPERSION DISCIPLINAIRE DU CORPS}

Le corps, comme objet d'étude philosophique, est très particulier, car cet objet n'est pas spécifiquement philosophique comme le sont, par exemple, les prédicats pour la logique ou le sens du vécu pour la phénoménologie. Objet d'autres disciplines, le corps oblige les philosophes à dialoguer inévitablement avec les autres sciences, qu'elles soient « humaines » ou non, comme la psychologie, la médecine, la neurologie, la biologie, l'informatique... et avec d'autres philosophies, de courants divers, non centrées directement sur le corps comme c'est, entre autres, le cas de la

\footnotetext{
${ }^{1}$ Nous reprenons ici, s'agissant d'expliciter la philosophie du corps, partiellement un texte que nous avons publié en 2008 dans Clinique du sport et des pratiques physiques aux Presses Universitaires de Nancy.

${ }^{2}$ Le terme " énactive " renvoie au concept de l'énaction énoncé par le philosophe Varela et qui signifie l'action incarnée.
} 
philosophie analytique. Pour ne citer qu'un seul exemple, l'analyse fine de la relation de l'identité entre le corps et l'esprit a ouvert des débats parmi les phénoménologues et les logiciens, les premiers s'interrogent davantage sur le lien entre l'esprit et le corps et les seconds s'interrogent sur le concept de l'identité entre ces deux substances.

De manière générale, les philosophes actuels du corps reconnaissent - au moins depuis les premiers phénoménologues du début du XXème siècle - que le corps est double, Leib et Körper, ce que nous désignons dans ce qui suit par l'expression de " corps sujet/objet ». Or, la philosophie du corps intéresse d'autres courants de la philosophie contemporaine que la seule phénoménologie et plus spécialement, la philosophie de l'esprit, la philosophie des sciences et la philosophie morale. Grosso modo, la philosophie du corps se subdivise en trois branches. D'abord, la philosophie de l'action qui se distribue en deux domaines, l'éthique corporelle et l'hédonisme. L'éthique (Marzano ${ }^{3}$, Nussbaum, Ogien,...) décrit les normes corporelles contemporaines. Les questions qu'elle pose sont diverses comme savoir jusqu'où peut aller la sexualité, s'il y a une représentation spéciale du corps dans la pornographie, ce qu'est le corps amoureux... L'hédonisme, essentiellement représenté aujourd'hui par le philosophe Michel Onfray, va, par contre, s'interroger plus spécialement sur la façon de rechercher la jouissance au travers les différents plaisirs du corps. Le corps est devenu le moyen d'accéder aux divers plaisirs par le désir défini comme une décharge, un excès et non comme un manque (Onfray ${ }^{4}$ ).

Ensuite, évoquons la philosophie des sciences humaines. II s'agit globalement des philosophes qui vont examiner la connaissance du corps dans les sciences de l'homme, sous la forme d'une épistémologie générale (Andrieu), mais aussi sous la forme d'épistémologies spéciales (Butler). L'épistémologie générale va se poser, entre autres choses, les questions suivantes : Comment définir le corps ? Que peut-on connaître du corps ? Comment connaître le corps comme subjectivité incorporée ? Quelle(s) science(s) peut (peuvent) étudier l'objet « corps » ? En plus de cette épistémologie générale, il existe également des épistémologies spéciales, notamment, celle du sexe et du genre. On attribue généralement à Judith Butler (1990) le fait d'avoir souligné la construction sociale et non biologique du genre et la distinction entre le sexe et le genre. Or, historiquement, Simone de Beauvoir dans le Deuxième sexe posait déjà cette question en se demandant ce qu'est une femme indépendamment de son sexe. A la même époque que Judith Butler, Elisabeth Badinter s'interrogeait sur la définition du genre masculin dans XY (1992). La philosophie du sexe et du genre est aujourd'hui institutionnalisée, surtout dans le monde anglosaxon, même si elle existait déjà en filigrane en philosophie contemporaine avant même son acte de naissance dans les années quatre-vingt-dix, avec le livre de Judith Butler Gendler Troubles, Feminism and the Politics of Subversion.

Pour terminer, envisageons la philosophie de l'identité entre l'esprit et le corps. Sous cette appellation, nous englobons à la fois la phénoménologie et la philosophie de l'esprit. En effet, la phénoménologie qui étudie le sens du vécu croise conceptuellement d'une manière ou d'une autre la philosophie de l'esprit qui, par définition, s'interroge sur l'esprit, autrement dit sur le vécu en première personne. Nous proposons de subdiviser la philosophie de l'identité de l'esprit/corps en trois sous-sections philosophiques essentielles à l'époque actuelle : la phénoménologie, la philosophie cognitive et l'énaction.

\footnotetext{
${ }^{3}$ Marzano, M. (2010). L'Ethique appliquée. Paris : Puf (Que-sais-je ?).

${ }^{4}$ Onfray, M. (2000). Théorie du corps amoureux. Paris : Grasset.
} 
Par « la phénoménologie », il s'agit aussi bien, pour nous, des héritiers de la phénoménologie que nous qualifions de "phénoménologie traditionnelle " (Barbaras, Giovannangeli, Seron), car ceux-ci sont conceptuellement plus proches des fondateurs du courant phénoménologique et sont des continuateurs des pensées de Husserl, de Merleau-Ponty, et de Sartre que des phénoménologues quelque peu "dissidents ". Nous pensons pour ces derniers, particulièrement, à Natalie Depraz et à Véronique Havelange dont les travaux sont des "échanges " avec la philosophie cognitive et plus spécialement, avec le paradigme de l'énaction. La philosophie cognitive désigne les chercheurs qui s'intéressent à l'étude de la conscience à la lumière des sciences cognitives (Cleeremans ${ }^{5}$, Jacob, Pacherie ${ }^{6}, \ldots$ ) alors que "l'énaction " renvoie à une branche particulière des sciences cognitives qui étudie le corps-chair dans une perspective nonsymbolique, incarnée et émergeante ( to enact »).

Cette subdivision est, comme nous l'avons annoncé, non exhaustive, mais cependant elle est suffisante pour laisser apparaître qu'il y a bien une philosophie du corps, malgré la diversité des courants et malgré toutes les nuances entre les différents penseurs. Cette multiplicité des courants s'éclaire par ce que Bernard Andrieu a appelé déjà au début des années quatre-vingt-dix le concept de " corps dispersé » en philosophie des sciences humaines. En substance, ce philosophe nous dit que le corps, comme objet d'étude en sciences humaines, est un objet multiple parce que celui-ci est divisé en autant de disciplines qui étudient celui-ci. Comme l'écrit Bernard Andrieu', le corps est divisé " parce que nous l'observons de manière externe en élaborant des modèles. » En ce sens, le corps étudié par le psychologue, par exemple, diffère du corps analysé par l'anthropologue ou du corps examiné par le philosophe. Selon Bernard Andrieu, la différence méthodologique qui existe entre les sciences impliquerait donc une différence conceptuelle.

Ce concept de " dispersion corporelle ", selon nous, permet d'expliciter la philosophie du corps d'aujourd'hui. En effet, même si tous les courants que nous avons décrits étudient le corps, tous font référence à une conception spécifique du corps. Les courants de l'éthique, de I'hédonisme, de l'épistémologie, de la philosophie du sexe et du genre, de la phénoménologie, de la philosophie cognitive et de l'énaction correspondent respectivement à la conceptualisation du corps suivante : le corps juridique, le corps affecté, le corps " recherché », le corps sexuel et sexué, le corps chair et vécu et enfin, le corps conscient et situé. En effet, pour le dire d'un mot, en philosophie de l'action, l'éthique s'intéresse au corps comme sujet/objet de droit et de devoirs et I'hédonisme se concentre davantage sur l'étude du corps affecté. En philosophie des sciences humaines, la philosophie du sexe et du genre s'interroge sur le corps sexuel et sexué tandis que l'épistémologie générale se demande ce qu'est un corps dans les différentes sciences humaines. Pour terminer, la philosophie de l'identité esprit/corps décrit le corps à la fois, chair et vécu (la phénoménologie qui questionne la conscience au sens philosophique), chair et conscience (la philosophie cognitive qui s'interroge sur la conscience psychologique), chair et vie (la philosophie énactive qui étudie le corps " situé "). Comme en sciences humaines, la philosophie du corps se disperse conceptuellement, car elle utilise des méthodes différentes pour étudier le corps. Par

\footnotetext{
${ }^{5}$ Cleeremans, A. (1993). Mechanisms of Implicit Learning: Connectionist Models of Sequence Processing. Cambridge, MA : MIT Press.

${ }^{6}$ Pacherie, E. (2002). Les consciences. Pour la Science, 302, 22-25.

${ }^{7}$ Andrieu, B. (1993). Le corps dispersé. Une histoire du corps au XXème siècle. Paris : L'Harmattan, p. 394.
} 
exemple, la méthode phénoménologique $\left(\operatorname{Seron}^{8}\right)$ diffère de la méthode de la philosophie cognitive (Pacherie ${ }^{9}$ ).

\section{LE CORPS TRANSDISCIPLINAIRE, MULTIPLE ET UN}

Les courants de pensée en philosophie du corps divergent, mais dans le même temps, ils sont intimement liés, souvent ils s'entrelacent, ils s'entrecroisent de multiples manières. Pour commencer, comme nous l'avons déjà évoqué plus haut, certains chercheurs de différentes écoles de pensée ont vu la nécessité de "dialoguer " (Depraz ${ }^{10}$, Cleeremans ${ }^{11}$... font, par exemple, dialoguer la philosophie de l'esprit et la phénoménologie). Ensuite, de plus en plus de travaux transdisciplinaires sont aujourd'hui publiés (ce qu'ont fait, notamment, Berthoz et Petit ${ }^{12}$ ). En outre, les diverses approches partagent un même objet d'étude (le corps). Les différents courants philosophiques permettent de rendre compte d'un point de vue possible sur le corps et l'ensemble de ces points de vue aide à expliciter la complexité de la définition du corps pour converger vers ce qu'on peut appeler une philosophie du corps.

L'apparente contradiction entre les nombreuses écoles de pensée et une branche spéciale de la philosophie qui serait la philosophie du corps peut s'expliquer mutatis mutandis par ce que François Dagognet ${ }^{13}$ disait de l'objet corps qui est à la fois multiple et un. C'est parce qu'il est divers et un que le corps comme objet d'étude est dispersé et unifié au regard des recherches pluridisciplinaires comme tentatives d'unification de la dispersion et des dialogues entre les divers courants philosophiques. Le corps est doublement multiple (au sein des sciences humaines et à l'intérieur même de la philosophie) et un [ifiable] de diverses manières en ce qu'une unification est possible entre plusieurs branches de la philosophie, entre une branche de la philosophie et une autre science humaine ou encore entre une approche philosophique et une science naturelle...

Pour résumer le paysage conceptuel de la philosophie du corps, nous proposons le tableau synoptique suivant qui reprend les différents courants et les philosophes contemporains du corps, selon les subdivisions évoquées plus haut.

\begin{tabular}{|c|c|c|c|c|c|c|}
\hline \multicolumn{7}{|c|}{ Paysage conceptuel de la philosophie du corps au XXlème siècle } \\
\hline \multicolumn{2}{|c|}{ Philosophie de l'action } & \multicolumn{2}{|c|}{$\begin{array}{l}\text { Philosophie des sciences } \\
\text { humaines }\end{array}$} & \multicolumn{3}{|c|}{$\begin{array}{l}\text { Philosophie de l'identité } \\
\text { esprit/corps }\end{array}$} \\
\hline $\begin{array}{l}\text { Éthique } \\
\text { Corps } \\
\text { sujet/objet de } \\
\text { droit et de } \\
\text { devoirs }\end{array}$ & $\begin{array}{l}\text { Hédonisme } \\
\text { Corps sujet/ } \\
\text { objet affecté }\end{array}$ & $\begin{array}{l}\text { Epistémologie } \\
\text { Corps sujet/ } \\
\text { objet d'étude }\end{array}$ & $\begin{array}{l}\text { Philosophie du } \\
\text { sexe et du } \\
\text { genre }{ }^{14} \\
\text { Corps sujet/objet } \\
\text { sexuel et sexué }\end{array}$ & \begin{tabular}{l|} 
Phénomén \\
ologie \\
Corps \\
sujet/objet \\
vécu
\end{tabular} & \begin{tabular}{|l} 
Philosophie \\
cognitive \\
Corps \\
sujet/objet \\
conscient
\end{tabular} & $\begin{array}{l}\text { Enaction }^{15} \\
\text { Corps } \\
\text { sujet/objet } \\
\text { situé }\end{array}$ \\
\hline
\end{tabular}

\footnotetext{
${ }^{8}$ Seron, D. (2001). Introduction à la méthode phénoménologique. Bruxelles : De Boeck Université.

${ }^{9}$ Pacherie, E. (2002). Les consciences. Pour la Science, 302, 22-25.

${ }^{10}$ Depraz, N. (2008). Lire Husserl en phénoménologue. Idées directrices pour une phénoménologie (I). Paris : Puf.

${ }^{11}$ Cleeremans, A. (1993). Mechanisms of Implicit Learning: Connectionist Models of Sequence Processing. Cambridge, MA : MIT Press.

12 Berthoz, A., et Petit, J.-L. (2006). Phénoménologie et physiologie de l'action. Paris : Odile Jacob.

${ }^{13}$ Dagognet, F. (1992). Le corps multiple et un. Paris : Les Empêcheurs de Penser en Rond.

14 Nous renvoyons ici le lecteur à Chalmers qui donne des informations sur son site concernant divers travaux des philosophes contemporains en philosophie du genre.

${ }^{15}$ Nous renvoyons notamment au lien suivant : http://www.arco.asso.fr/.
} 


\begin{tabular}{|c|l|l|l|l|l|l|}
\hline M. Marzano & M. Onfray & B. Andrieu & S. Agacinski & N. Depraz & D. Chalmers & H. Dreyfus \\
\hline R. Ogien & & A. Atlan & E. Badinter & J-L. Petit & $\begin{array}{c}\text { A. } \\
\text { Cleeremans }\end{array}$ & $\begin{array}{c}\text { V. } \\
\text { Havelange }\end{array}$ \\
\hline & & F. Dagognet & J. Butler & & S. Gallagher & A. Noé \\
\hline & & V. Despret & D. Haraway & & P. Jacob & $\begin{array}{l}\text { E. } \\
\text { Thompson }\end{array}$ \\
\hline & & J-N. Missa & B. Preciado & & E. Pacherie & J. Stewart \\
\hline & & I. Queval & P. Bruckner & & & \\
\hline & & & P. Sastre & & & \\
\hline
\end{tabular}

Table 1: Paysage conceptuel de la philosophie du corps au XXIème siècle

Comme nous l'avons décrit dans ce qui précède, la philosophie du corps se développe aujourd'hui notamment autour de trois grands axes :

1) la philosophie de l'action avec Onfray pour l'hédonisme et Marzano, entre autres, pour l'éthique,

2) la philosophie des sciences humaines, notamment, avec Andrieu et Dagognet, et enfin,

3) la philosophie de l'identité de l'esprit/corps qui comporte trois sous-axes distincts (la phénoménologie, la philosophie cognitive et la philosophie de l'énaction) qui s'entrelacent et qui sont représentés par des chercheurs aussi divers que Cleeremans, Depraz, Gallagher, Stewart,... Institutionnalisée (Andrieu, Marzano), en marge (Onfray) ou fondée tout récemment (Havelange, Thompson,...), la philosophie du corps est, pour paraphraser François Dagognet, " multiple et une ", car elle explore de diverses manières ce qu'est fondamentalement le corps.

\section{CONCLUSION}

Nous avons dans ce qui précède donné un aperçu de la philosophie contemporaine du corps. Nous avons souligné la diversité des études sur le corps comme autant de points de vue possibles sur cet objet spécifique et dans le même temps, nous avons mis en évidence l'unité de cette pensée qui considère un objet difficile à cerner sous un seul angle. Le corps est un objet d'étude dans le même temps « multiple et un ».

\section{BIBLIOGRAPHIE}

ANDRIEU, Bernard. La peur de l'orgasme. Strasbourg : Le murmure : 2013.

. L'autosanté. Vers une médecine réflexive. Paris : Armand Colin, 2012.

Les avatars du corps. Hybridités et somatechnies. Paris : Liber, 2011.

Philosophie du corps. Expériences, interactions et écologie corporelle. Paris : Vrin, 2010.

. Le monde corporel. De la constitution interactive du soi. Lausanne : L'Age d'Homme, 2010.

. Devenir hybride. Nancy : Pun, 2008.

Mutations sensorielles. Nancy : Le Mort-Qui-Trompe, 2008. 
Le seul crime réel de l'homme serait de troubler l'ordre de la nature, Sade. Nantes : Editions Pleins Feux, 2007.

Dictionnaire du corps. Paris : Cnrs, 2006.

. La nouvelle philosophie du corps. Paris : Erès, 2002.

. Le corps dispersé. Une histoire du corps au XXème siècle. Paris : L'Harmattan, 1993.

ATLAN, H. L’Utérus artificiel. Paris : Seuil, 2005.

BERTHOZ, A. ; PETIT, J.-L. Phénoménologie et physiologie de l’action. Paris : Odile Jacob, 2006.

BUTLER, J. Défaire le genre. Paris : Editions Amsterdam, 2012.

. Trouble dans le genre. Pour un féminisme de la subversion. Paris : La Découverte, 2005.

CHALMERS, D. Philosophy of Mind. Classical and Contemporary Readings. Oxford : University Press, 2002.

CLEEREMANS, A. Mechanisms of Implicit Learning: Connectionist Models of Sequence Processing. Cambridge, MA : MIT Press, 1993.

DAGOGNET, F. Le corps. Paris : PUF, 2008.

. Pour une philosophie de la maladie. Paris : Textuel, 1996.

. La peau découverte. Paris : Les Empêcheurs de Penser en Rond, 1993.

. Le corps multiple et un. Paris : Les Empêcheurs de Penser en Rond, 1992.

DE BEAUVOIR, S. Le Deuxième sexe. Paris : Gallimard, 1949.

DE SAINT-POINT, V. Manifeste de la femme futuriste. Paris : Mille et une Nuits, 2005.

DEPRAZ, N. Lire Husserl en phénoménologue. Idées directrices pour une phénoménologie (I). Paris : PUF, 2008.

Comprendre la phénoménologie. Une pratique concrète. Paris : Armand Colin, 2006.

DESPRET, V. Ces émotions qui nous fabriquent. Paris : Les Empêcheurs de Tourner en Rond, 1999.

DORLIN, E. Sexe, genre et sexualités : introduction à la théorie féministe. Paris : PUF, 2008.

GALLAGHER, S. How the Body Shapes the Mind. Oxford : Oxford University Press, 2005.

GIOVANNANGELI, D. Le retard de la conscience. Husserl, Sartre, Derrida. Bruxelles : Ousia, 2001.

HARAWAY, D. Manifeste cyborg et autres essais : sciences - fictions-féminismes. Paris : Editions Exils, 2007.

HAVELANGE, V. ; LENAY C. ; STEWART J. Les représentations : mémoire externe et objets techniques, Intellectica, n. 35, p. 115-131, 2003.

LUTZ, A.; THOMPSON, E. Neurophenomenology. Integrating Subjective Experience and brain Dynamics in the Neuroscience of Consciousness, Journal of Consciousness Studies, n. 10, p. 9-10, 31-52, 2003.

MARZANO, M. Légère comme un papillon. Paris : Grasset, 2011.

. L’Éthique appliquée. Paris : PUF, 2010.

Philosophie du corps. Paris : PUF, 2007.

Dictionnaire du corps. Paris : PUF, 2007.

Malaise dans la sexualité. Le piège de la pornographie. Paris : Lattès, 2006.

METIVIER F. ; OVIDIE. Sexe Philo. Paris : Editions Bréal, 2012.

ONFRAY, M. Féeries anatomiques. Paris : Grasset, 2003. 
JOLY (2015)

. L'invention du plaisir. Paris : Grasset, 2002.

. Théorie du corps amoureux. Paris : Grasset, 2000.

. L'art de jouir. Paris : Grasset, 1991.

PACHERIE, E. Les consciences, Pour la Science, n. 302, p. 22-25, 2002.

PRECIADO, B. Pornotopie. Playboy et l'invention de la sexualité multimédia. Paris : Climats, 2011.

Manifeste contra-sexuel. Paris : Balland, 2011.

. Testo Junkie : sexe, drogue et biopolitique. Paris : Grasset, 2008.

QUEVAL, I. Le corps aujourd'hui. Paris : Gallimard, 2008.

SASTRE, P. Pour en finir avec le féminisme. Paris : La Musardine, 2009.

SERON, D. Introduction à la méthode phénoménologique. Bruxelles : De Boeck Université, 2001.

STENGERS, I. ; DESPRET, V. Les faiseuses d'histoires. Que font les femmes à la pensée ? Paris : Les empêcheurs de penser en rond/La découverte, 2011.

VARELA, F. J. ; PETITOT, J. ; PACHOUD, B. ; ROY, J-M. Naturaliser la phénoménologie. Paris : CNRS, 2002. 